\title{
Combined Auditory and Tragal Stimulation Headphone Effectively Increases Vagal Tone and Improves Short-Term Cognition
}

\author{
${ }^{1}$ Century High School, USA \\ ${ }^{2}$ Mayo Clinic Department of Cardiology, USA \\ ${ }^{3}$ Mayo Clinic Department of Pediatrics and Adolescent Medicine, USA \\ ${ }^{4}$ Mayo Clinic Department of Biomedical Engineering, USA
}

Hemanth 0 Asirvatham ${ }^{1}$, Jennifer A Mears BS ${ }^{2}$, Samuel J Asirvatham MD*2,3,4

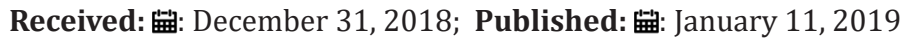

*Corresponding author: Samuel J Asirvatham, Mayo Clinic Department of Biomedical Engineering, USA

\section{Abstract}

The autonomic nervous system is responsible for many psychological and physiological phenomena. Music is known to influence the autonomic nervous system and provide many beneficial effects in both the body and the mind. The vagus nerve has also been stimulated to invoke similar beneficial effects. Since this procedure is invasive, the tragus nerve, which is the only input to the vagus, is ideal for autonomic modulation. While this effect has been demonstrated alone, the combination of music and tragal stimulation, two very similar interventions, has not been studied yet.

Hypothesis: A modified headphone with combined stimulatory electrodes on the tragus will safely and effectively stimulate the vagus nerve while listening to music. Such combined stimulation will improve autonomic tone, cognition, and mood.

Methods: Autonomic functions such as heart rate, blood pressure, heart rate variability, and electrodermal activity as well as cognition and emotions were recorded during various interventions. These interventions included music (major and minor chords), tragal simulation alone, and the combination of both.

Results: All participants completed the experiment without any adverse effects. There was a statistically significant decrease in heart rate $(p=0.038)$ and increase in heart rate variability $(p=0.001)$ in response to tragal stimulation. The heart rate variability increase was more pronounced in females indicating previously unreported phenomena. Cognition improved significantly $(\mathrm{p}=0.008)$ with tragal stimulation and music combined.

Conclusion: A novel integrated headphone stimulatory device can be used safely and effectively to improve and modulate autonomic tone and cognition while enjoying a musical experience.

Keywords: Tragus; Vagus; Autonomic Tone; Headphones; Music; Cognition; Heart Rate Variability; Sudden Death

Abbreviations: ANS : Autonomic Nervous System; CNS : Central Nervous System; BP: Blood Pressure; HR : Heart Rate; HRV : Heart Rate Variability; EDA : Electrodermal Activity; ECG : Electrocardiogram ; DSST : Digit Symbol Substitution Test

\section{Introduction}

One of the few major afferent routes to the central nervous system (CNS) is the vagus nerve [1]. While there are many different outputs, including motor output, from the CNS, afferent nerves tend to be processed at the brainstem or spinal cord level. An exception is the vagus nerve, and as a result of this unique vantage point to the CNS and through it to various parts of the body, stimulation and modulation of the vagus nerve afferents have become an area of burgeoning research with multiple implications for health and disease [2]. The vagus nerve has been implicated predominantly for cardiovascular health, including the prevention of sudden death[3]. Vagal stimulation not only decreases heart rate (HR) and blood pressure (BP) but also importantly creates a more positive healthy profile for heart rate variability (HRV). Indeed, vagal stimulation has been shown to acutely treat arrhythmias such as atrial fibrillation $[4,5]$. A potential major effect is reducing the chance of sudden death through the tragal effects on HRV [6]. By virtue of the afferent 
input to the CNS, vagal stimulation has also produced benefits in depression, cognition, and certain degenerative disorders $[7,8]$. However, the promise of such treatments to help patients with various disorders has fallen short of expectations. The reasons for this are varied but may include a lack of understanding of the optimal stimulatory sequences for each disease. Primarily, however, is the need for invasive procedures with known morbidity in order to employ this therapy even when studying how to best use it [9].

The first extracranial branch of the vagus nerve involves peripheral entry through the tragus and external auditory meatus. Free nerve fibers can be traced to the vagus from these locations. Also, due to the relatively superficial placement of the nerve fibers, there is a high degree of noninvasive accessibility to the vagus through these fibers. However, there are a great deal of unknowns with regard to the high impact potential of tragus and external auditory meatus vantage point stimulation for cardiovascular health and other diseases. Investigating such unknowns could reveal a superior form factor that patients would be more likely to use over longer periods of time, a superior method to test different patterns and degrees of stimulation to identify optimal parameters, and more comfortable and usable designs for stimulation. Furthermore, experimentation could reveal the interactions between tragal stimulation and sound, specifically concerning auditory noise, stimulation, and music $[10,11]$.

\section{Hypothesis}

A modified earphone with combined stimulatory electrodes on the tragus can safely and effectively stimulate the vagus nerve while listening to music. Such a combined stimulation will improve autonomic tone, cognition, and mood.

\section{Methods}

Our study consisted of two phases (Figure 1):

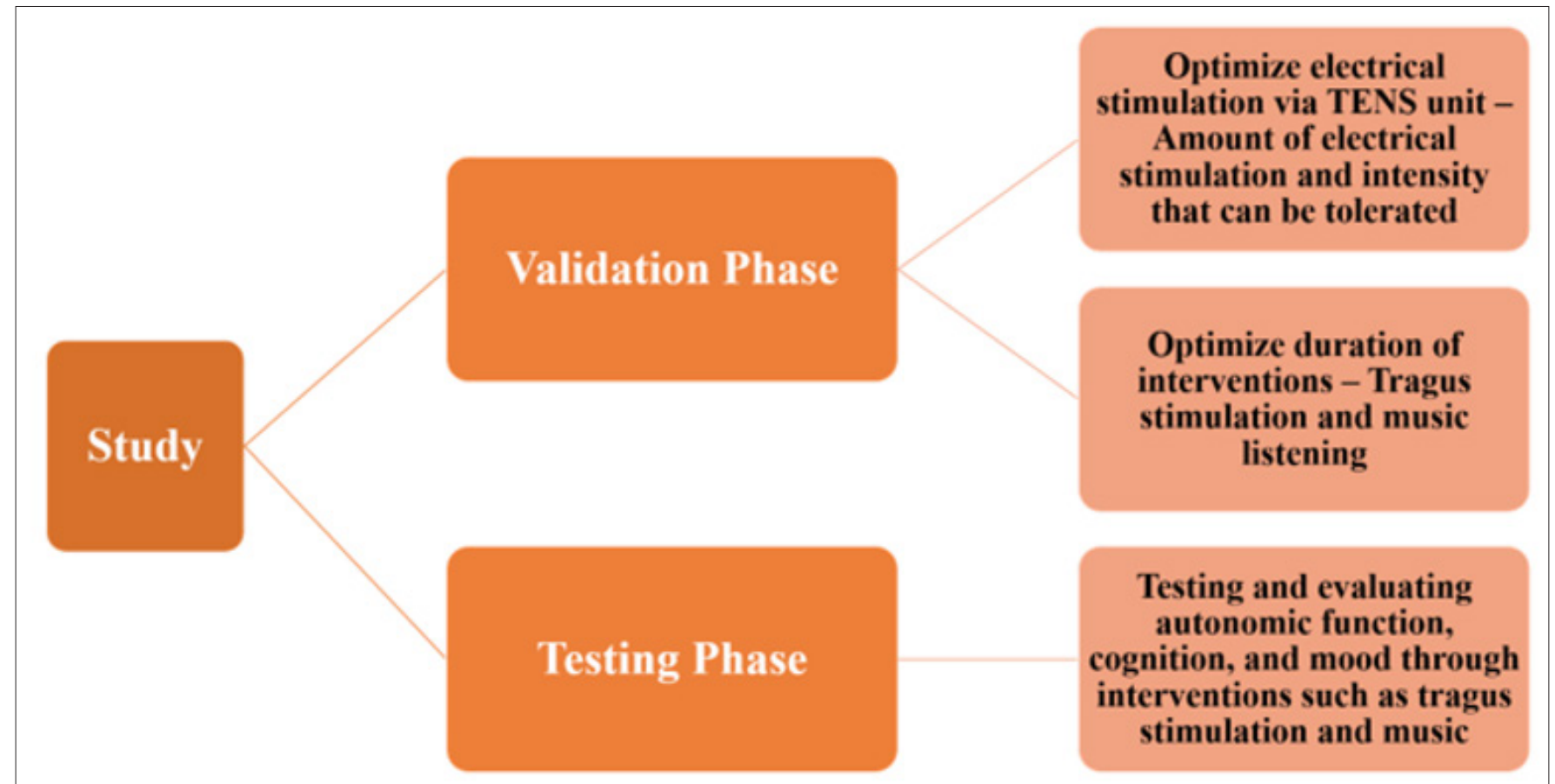

Figure 1: Diagram of different phases in methodology.

a) Validation Phase: The amount of electrical stimulation and intensity to produce a tolerable tragal stimulation via a TENS unit was optimized. The duration was also optimized based on previous studies.

b) Testing Phase: 19 adult volunteers were recruited after informed consent. Each participant served as his/her own control. The autonomic function of each participant was measured by evaluating his/her HR,BP,HRV, and electrodermal activity (EDA). These initial baseline measurements were followed by measurements after listening to music, after undergoing tragus stimulation using TENS unit for 5 minutes, and after tragus simulation combined with music listening.

\section{Tragal Stimulation}

Tragal stimulation was achieved by connecting a portable TENS unit (TENS 7000冈) to the tragus of the participant using ear clips attached to the tragus of both ears. Tolerable settings obtained from the validation testing were used to achieve adequate tragus stimulation. This included a pulse width of $200 \mu$ s, pulse rate of $50 \mathrm{~Hz}$, and an intensity of 2 . After 5 minutes of tragus stimulation, autonomic testing was completed, and music was added (Figure 2). 


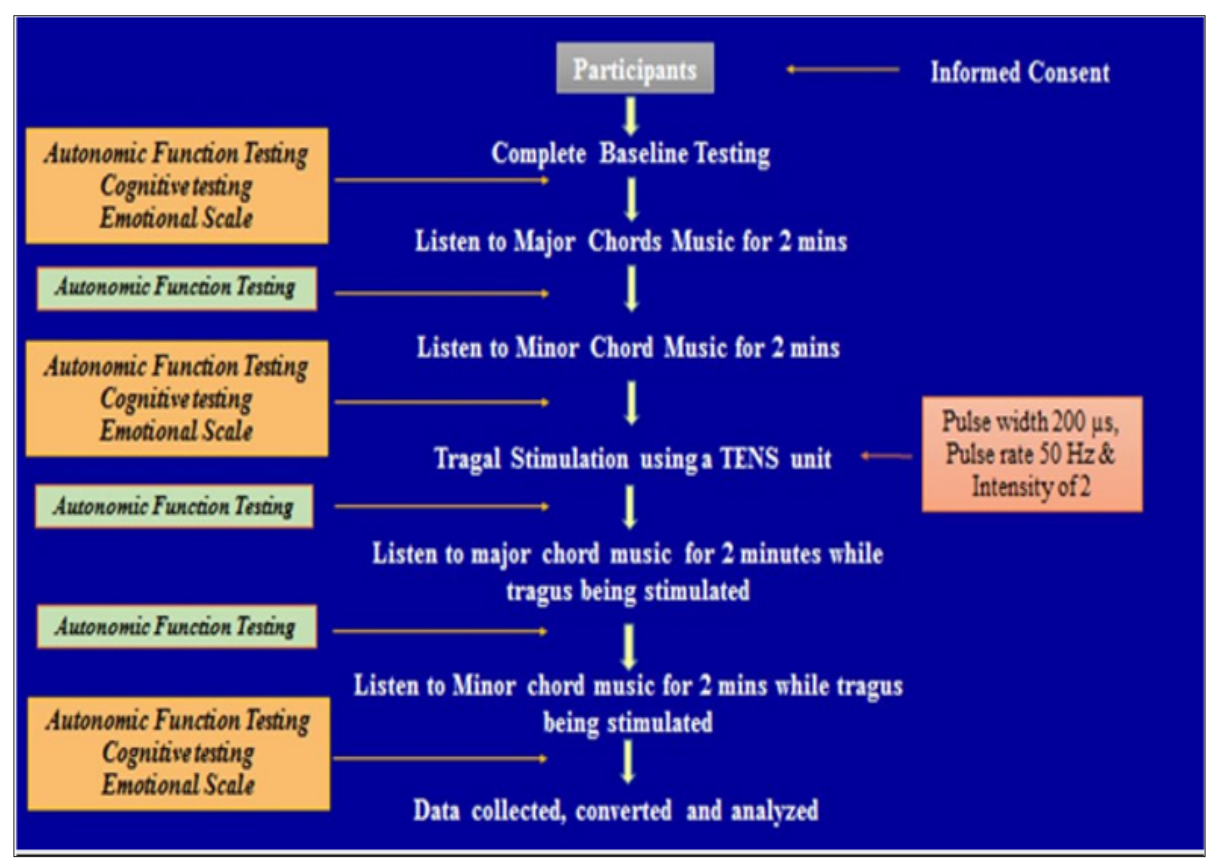

Figure 2: Flowchart indicating different components and treatments of methodology in application on a single individual. The application of treatments on the subject is in chronological order.

\section{Music}

Since it has been shown that music of varying tempo results in varied physiological responses [12], the participants were asked to listen to 2 types of music:

a) Major chords, fast tempo, invigorating music for 2 minutes

b) Minor chords, slow tempo, calming music for 2 minutes.

\section{Evaluation of Autonomic Response}

Since the autonomic nervous system (ANS) is a complex system, there is no single test that can evaluate its functions [13]. However, there are a variety of invasive and noninvasive tests being used to evaluate the functions of the ANS. Due to availability and simplicity on testing participants out of a laboratory conditions, we chose to measure the following to assess their autonomic response to tragus stimulation and music.

Blood Pressure: A digital BP monitor was used to measure the participant's BP several times during the study.

Heart Rate: A portable electrocardiogram (ECG) monitoring system called Alivecor冈 was used. Participants were taught how to hold it during the study, and the recordings were uploaded to the computer and analyzed.

Heart Rate Variability: Biological processes such as the HR are complex; they are never truly steady at rest. There are constant dynamic bidirectional changes in HR influenced by multiple factors including neural, hormonal, and mechanical systems. The beatto-beat fluctuation in HR is called HRV, and it is the most accurate reflection of the physiological factors influencing the normal rhythm of the heart. The electrical QRS complex in an ECG indicates a single heartbeat. The beat-to-beat time duration can be measured by measuring the time duration between 2 consecutive QRS complexes or simply by measuring the RR interval. It is well known that even at rest the RR interval is not constant but continually fluctuates around a mean value. Often overlooked when expressed as a mean value, the HRV is a measure of neurocardiac function [14]. Complex neural mechanisms are responsible for these fluctuations. Particularly, the interaction between sympathetic and parasympathetic nervous systems influences the variations in HR [15]. An optimal level of variability in the HR indicates the flexibility/adaptability of the heart functions. The higher a person's HRV is, the better his/her heart health. Reduced HRV usually correlates with higher cardiac mortality.

\section{HRV Measurement and Analysis}

The raw data from Alivecor囚 was translated into usable data using a Python program (Figure 3). This resulted in a large amount of data regarding the RR interval for each participant. In order to calculate the HRV, TIME-DOMAIN analysis was used. Time domain measures are the simplest to calculate and include the mean normal-to-normal intervals during the entire recording. There are many ways to obtain time domain measures such as SDNN, SDANN, rMSDD and pNN50. We chose to use the rMSDD method because it works for short-term recordings. rMSDD is the root mean square of successive differences between normal heartbeats. This is obtained by first calculating each successive time difference between heartbeats in milliseconds. Then, each of the values is squared, and the result is averaged before the square root of the total is obtained. The MSSD reflects the beat-to-beat variance in HR and is the primary time domain measure used to estimate the vagallymediated changes reflected in HRV. 


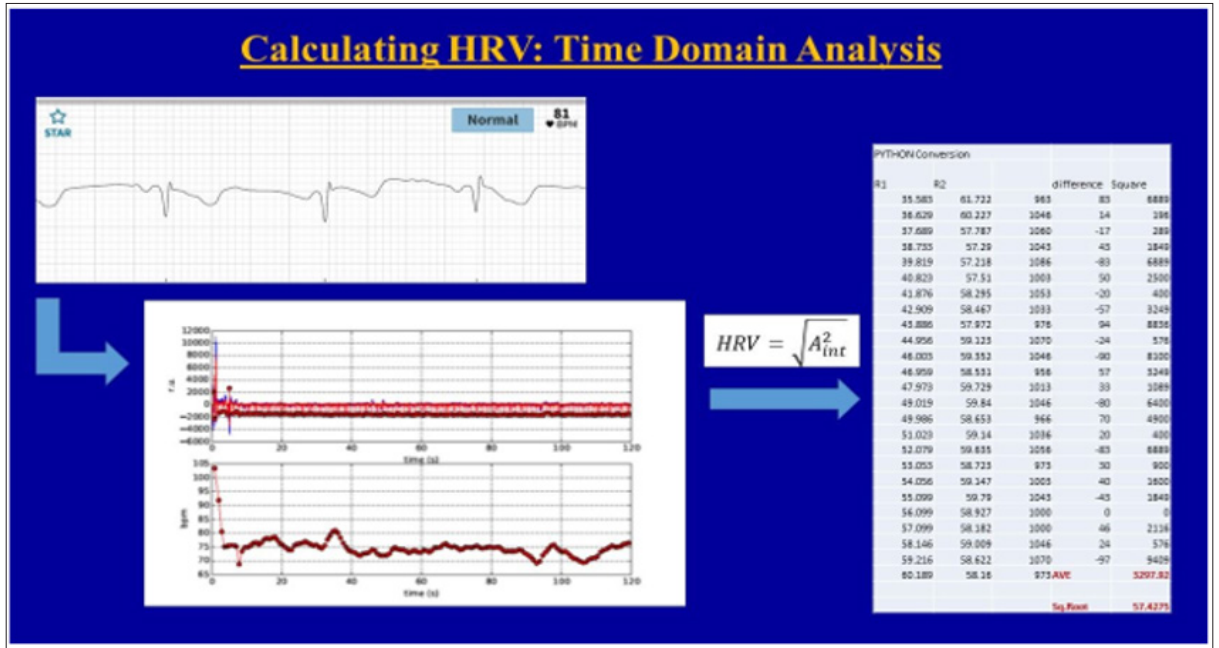

Figure 3: Diagram illustrates the process of analyzing HR variability. Data was initially obtained through Alivecor冈 software, and raw data values were found utilizing a Python program for conversion.

\section{Electrodermal Activity}

Formerly known as the galvanic skin response, the EDA is the term used to define autonomic changes in the electrical properties of the skin. The skin conductance is measured by applying an electrical potential between two points on the skin and measuring the current flow between them. The skin conductance is believed to vary with the amount of sweating, which is controlled by the sympathetic nervous system. The EDA is considered the most useful indicator of sympathetic arousal as it is the only autonomic psychophysiological variable that is not contaminated by parasympathetic activity [16].

\section{Results}

\section{Assessment of Cognition and Emotion}

Since the ANS plays a significant role in emotional and cognitive states, they were assessed before and after tragus stimulation.

Cognition: An online cognitive test for digit substitution which allowed for varying questions of equal difficulty was administered to the participants after instruction and two practice trials. The digit substitution test is a well-established test of cognition used clinically to measure cognitive functions such as memory and speed of processing. Other cognitive skills such as attention, graphomotor skills, and executive control can also be measured with this test.

Emotions: Both music and the ANS are known to influence emotions and therefore the emotional status of the participants was assessed using the self-reported PANAS scale at various times during the study [17]. The objective and self-reported parameters obtained were tabulated on an Excel program, and statistical analysis for variance, significant differences between groups, and effects were completed. There were 19 total participants in the study ( 8 males and 11 females). The participants ranged in age from 18 to 53 years old. A vital observation was that all participants were able to tolerate tragal simulation without any untoward effects including pain or discomfort.

\section{Autonomic Response-HR}

There was a decrease in the average HR of the participants with tragal stimulation when compared to their baseline, which was statistically significant $(\mathrm{p}=0.04)$ (Figure 4$)$. This trend indicates the possible cardioprotective effect of tragal stimulation.

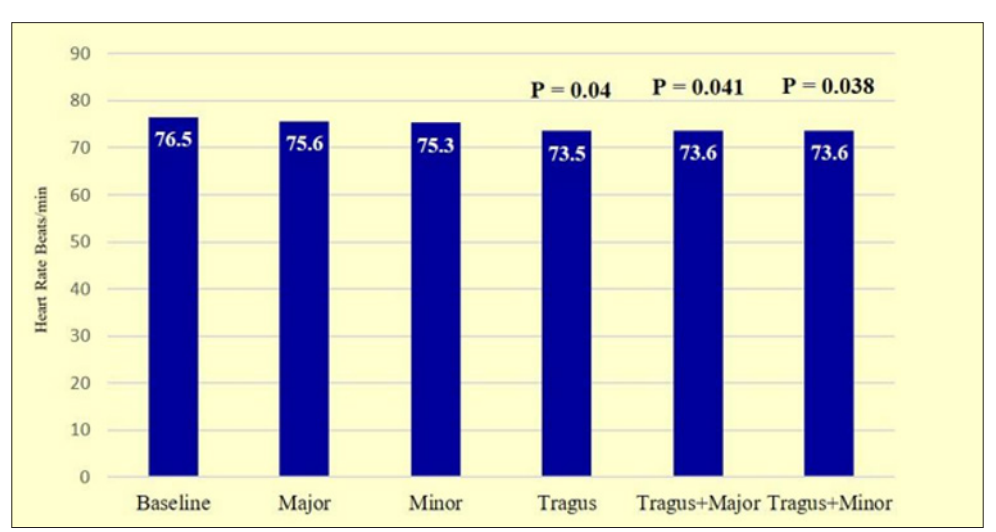

Figure 4: Bar graph illustrates a comparison of HR over the different treatments. A statistically significant decline $(p=0.04)$ was only observable in treatments utilizing tragal stimulation. 


\section{Autonomic Response - Changes in HRV}

In this pilot study, the HRV increased with tragal stimulation when compared to the baseline (Figure 5). This change was statistically significant $(\mathrm{p}=0.001)$. Increased HRV means an increased level of flexibility/adaptability of the heart functions, which further indicates the health of the heart. Tragal stimulation appears to be cardioprotective. Increased HRV is a potent marker for decreased cardi- ac events and sudden death and has multiple other positive health benefits. This study suggests that tragal stimulation can achieve increased HRV without pain or discomfort. Future design may include even stronger stimulations with a device that fits around the tragus, possibly cushioning the tragus, yet having a conductive material that can take the stimulatory pulse to the superficial branches of the vagus nerve. As a result, more potent benefits can be achieved.

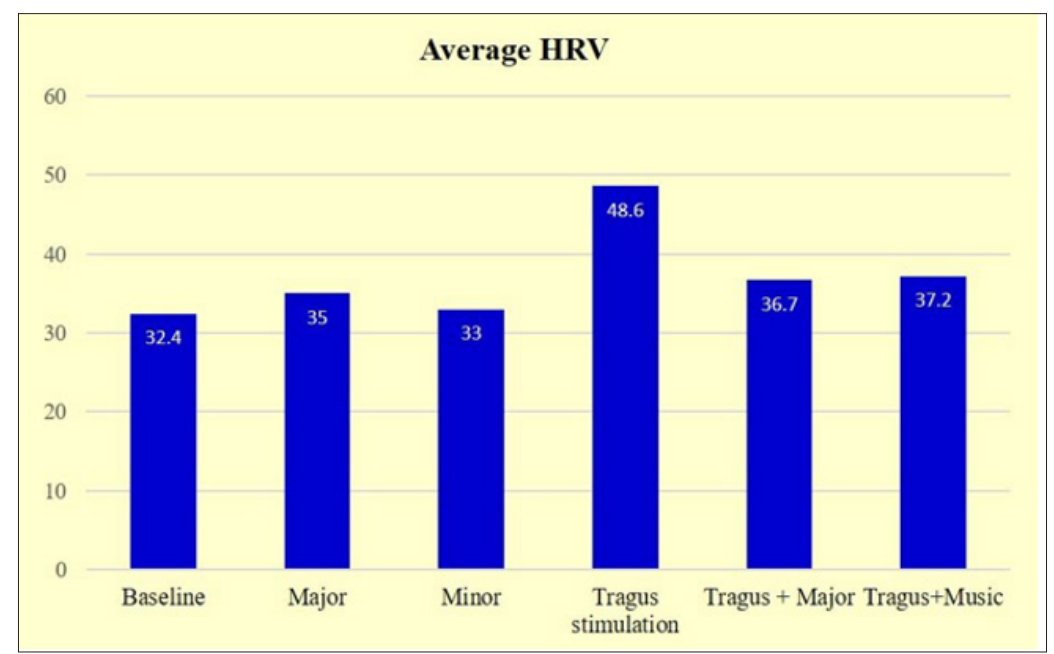

Figure 5: Graph illustrating HRV over the different treatments. A statistically significant increase $(p=0.001)$ in HRV was only observed under the treatment of tragal stimulation. The effect did not continue when music was added.

\section{Autonomic Response - Changes in BP and EDA}

The EDA and BP tests showed no major effect. Both measure long-term changes in autonomic tone and thus may only be affected with long-term tragal stimulation [18]. Longer exposure research must be done to determine any effects.

\section{Effects on Cognition}

The Digit Symbol Substitution Test (DSST) is used to measure cognitive functions such as attention and $\mathrm{m}$. The scores on the DSST significantly improved with tragal stimulation alone and with the addition of music in this study $(p=0.008)$ (Figure 6). This improvement cannot be attributed to better performance with practice as all participants were given adequate time to practice these tests prior to the study. In order to explore if the percent increase correlates with the baseline scores, the data was grouped with the baseline scores into lower third, middle third, and upper third and analyzed for changes. As seen in the graph below, the participants with a low baseline score appeared to have benefited the most from the interventions of tragal stimulation and music combined (Figure 7).

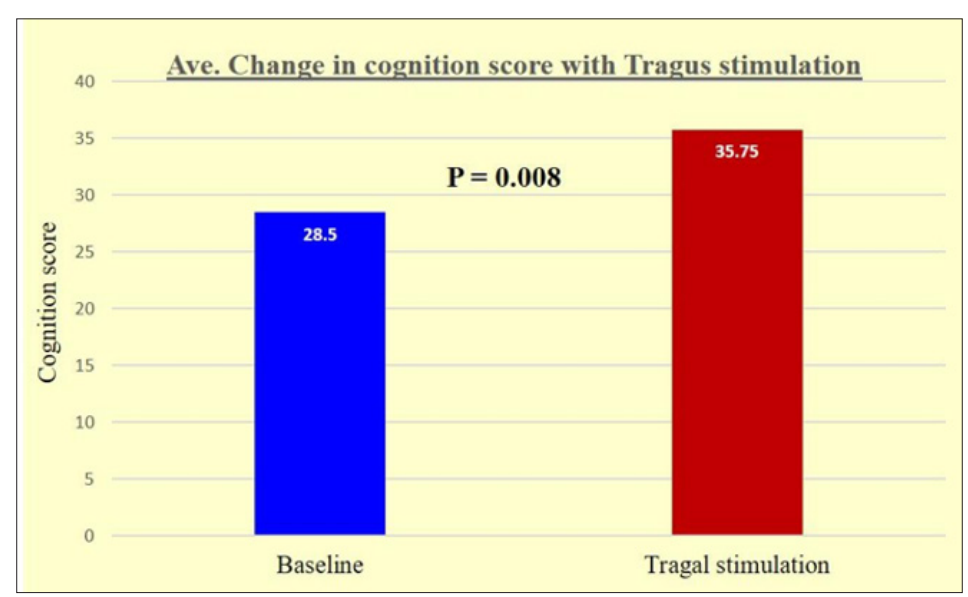

Figure 6: Graph illustrating cognitive performance on the DSST under the baseline and tragal stimulation treatments. There was a statistically significant increase $(\mathrm{p}=0.008)$ in performance with this treatment. 


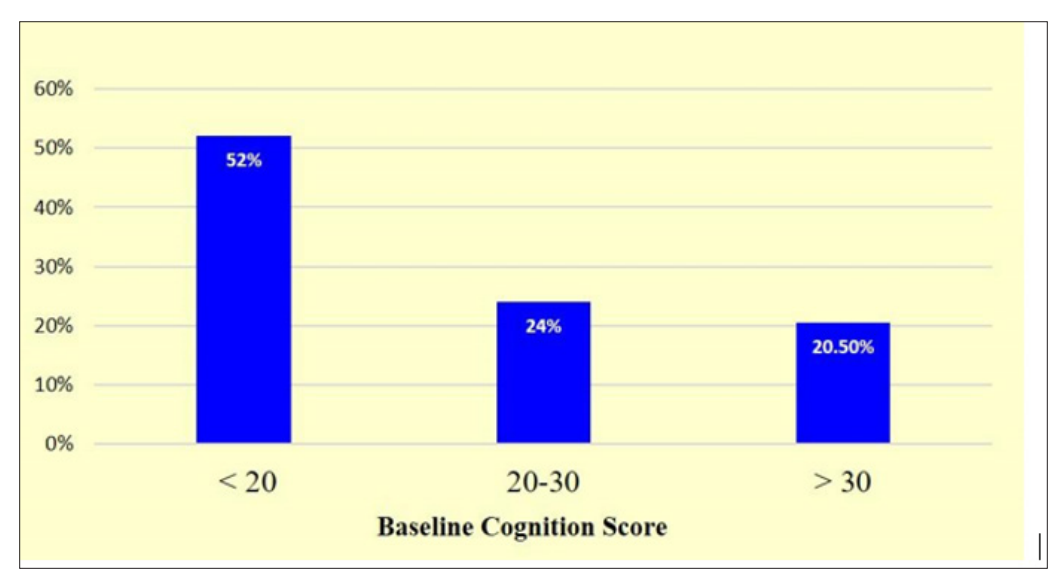

Figure 7: Graph illustrating percent improvement in cognitive scores based on initial cognitive performance. While all ranges of initial performance showed improvement, the greatest percent improvement was observed in those with the poorest initial performance.

\section{Discussion}

The ability of tragal stimulation to modulate HR and HRV is well supported by existing vagus nerve efferent. However, while direct stimulation of the vagus nerve has shown improvements in HR and HRV, the ability of tragal stimulation to achieve similar benefits while being comparatively non-invasive proves its viability over current methods [3]. The vagus nerve has a unique vantage point to affect cognitive functions in multiple ways it has efferent tracks going both to the ANS in the body as well as the brain. This has been used in the medical field to treat depression by placing devices that stimulate the vagus and improve afferent traffic to the brain for CNS benefits including mood and cognition. The vagus has been described as the "calming nerve," slowing the HR and with similar effects on the gastrointestinal tract and other involuntary muscle tracts. This may produce and mimic the sense of calmness, decreased anxiety, and increase the ability of an individual to focus on a particular intellectual task [12].

Usually, arousal and calming are mutually exclusive with certain factors like sympathetic stimulation increasing arousal, meditation, decreasing anxiety, and increasing focus. The vagus nerve, because of its varied yet cohesive afferent and efferent effects, may be unique in creating a best-case scenario for improving cognition. Students often listen to music while studying. This combined device may be suitable for getting the neuromodulator benefits along with the benefits and pleasure of listening to music. Other scenarios where decreased anxiety and increased focus could be of value could use a similar device like tragal stimulation without necessarily the music.

\section{Limitations}

Being a pilot study, this experiment had a relatively small number of participants. A pivotal study with larger number of participants will be needed to confirm these findings. Beyond this, the exact duration of each of the interventions used in this study was not known. Further experimentation could be used to reveal the optimal amount of treatment. Finally, while the safety and comfort were established with the validation phase, further studies are needed to explore the balance between optimizing the efficacy of the procedure while minimizing adverse effects or any possible discomfort that may arise.

\section{Implications}

This study revealed the profound potential of tragal stimulation. The overarching implication is that tragal stimulation, through its positive impact on the autonomic nervous system, can be used as a potentially preventative procedure to address sudden cardiac death by using a simple and noninvasive treatment. Other implications include the positive effect on cognition that tragal stimulation posed and how this can be used to help people with learning disabilities. Finally, the potential for the integration of music and tragal stimulation presents the possibility of an entirely new musical experience - one that is both enjoyable and healthy.

\section{Future Directions}

The results of this study reveal many potential paths for further exploration. Additional experimentation should be used to determine the optimal parameters of tragal stimulation, maximizing autonomic benefit while minimizing any adverse effects. Furthermore, the interesting results regarding the impact of tragal stimulation on cognition should be further studied in a separate trial. Similarly, the effect of tragal simulation on reducing the occurrence of sudden cardiac death should be analyzed in a trial of patients who already have defibrillators. Finally, the prototype device used in this experiment should be upgraded to analyze the potential for feedback tragal stimulation, which takes input from an individual's current autonomic state and uses that to modulate the tragal simulation parameters to best suit the circumstances.

\section{Conclusion}

A novel integrated headphone stimulatory device can be used safely and effectively to improve and modulate autonomic tone while enjoying a musical experience, affirming the hypothesis with the exception of an emotional change [19]. 


\section{References}

1. Blessing B, I Gibbins (2008) Autonomic Nervous System. Scholarpedia 3(7): 2787

2. Madhavan M, Christopher V DeSimone, Siva Mulpuru, Samuel J Asirvatham (2013) Renal nerve stimulation for the treatment of neurocardiogenic syncope. Cardiovasc Revasc Med 14(3): 172-173.

3. Van Leusden JW, R Sellaro, LS Colzato (2015) Transcutaneous Vagal Nerve Stimulation (tVNS): a new neuromodulation tool in healthy humans? Front Psychol 6: 102.

4. Sha Y, Scherlag BJ, Yu L, Sheng X, Jackman WM, et al. (2011) Low-level right vagal stimulation: anticholinergic and antiadrenergic effects. J Cardiovasc Electrophysiol 22(10): 1147-1153.

5. Yu L, Scherlag BJ, Li S, Fan Y, Dyer J, et al. (2013) Low-level transcutaneous electrical stimulation of the auricular branch of the vagus nerve: a noninvasive approach to treat the initial phase of atrial fibrillation. Heart Rhythm 10(3): 428-435.

6. (2014) Health ID Implantable Vagal Nerve Stimulator Cures Resistant Hypertension.

7. Ben Menachem E (2002) Vagus-nerve stimulation for the treatment of epilepsy. Lancet Neurol 1(8): 477-482.

8. Tisi G, Franzini A, Messina G, Savino M, Gambini O (2014) Vagus nerve stimulation therapy in treatment-resistant depression: a series report. Psychiatry Clin Neurosci 68(8): 606-611.

9. Rielo D (2015) Vagus Nerve Stimulation: Overview, Mode of Action, Technical Aspects.

10. Bernardi L, Porta C, Casucci G, Balsamo R, Bernardi NF, et al. (2009) Dynamic interactions between musical, cardiovascular, and cerebral rhythms in humans. Circulation 119(25): 3171-3180.

ISSN: 2574-1241

DOI: 10.26717/BJSTR.2019.13.002351

Samuel J Asirvatham. Biomed J Sci \& Tech Res

(C) This work is licensed under Creative Commons Attribution 4.0 License

Submission Link: https://biomedres.us/submit-manuscript.php
11. Valenti VE, Guida HL, Frizzo AC, Cardoso AC, Vanderlei LC, et al. (2012) Auditory stimulation and cardiac autonomic regulation. Clinics (Sao Paulo) 67(8): 955-958.

12. Thoma MV, Roberto La Marca, Rebecca Brönnimann, Linda Finkel, Ulrike Ehlert, et al. (2013) The effect of music on the human stress response. PLoS One 8(8): e70156.

13. Kapa S, KL Venkatachalam, SJ Asirvatham (2010) The autonomic nervous system in cardiac electrophysiology: an elegant interaction and emerging concepts. Cardiol Rev18(6): 275-284.

14. Van der Zwaag M, E Van den Broek, J Westerink (2011) Emotional and pyschological responses to tempo, mode, and percussiveness. Musicae Scientiae 15(2): 250-269.

15. Mc Craty R, F Shaffer (2015) Heart Rate Variability: New Perspectives on Physiological Mechanisms, Assessment of Self-regulatory Capacity and Health risk. Glob Adv Health Med 4(1): 46-61.

16. Kleiger RE, PK Stein, JT Bigger (2005) Heart rate variability: measurement and clinical utility. Ann Noninvasive Electrocardiol 10(1): 88-101.

17. Watson D, LA Clark, A Tellegen (1988) Development and validation of brief measures of positive and negative affect: the PANAS scales. J Pers Soc Psychol 54(6): 1063-1070.

18. Braithwaite J, Derrick G Watson, Robert Jones, Mickey Rowe (2015) A guide for analyzing electrodermal activity (EDA) and skin conductance response (SCRs) for physchological experimentation B.M.R.A. software.

19. Asirvatham SJ (2018) Cardiac electroporation: The promise of the unknown. J Cardiovasc Electrophysiol 29(4): 652-654.

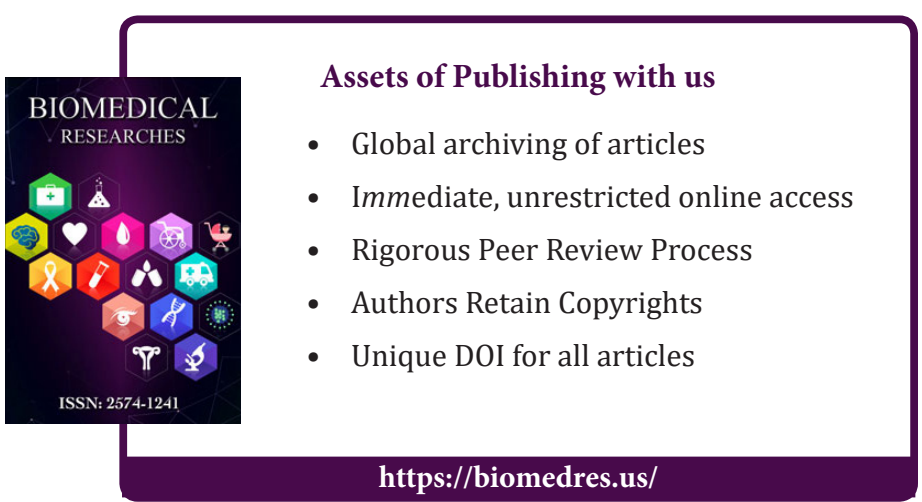

Cite this article: Hemanth 0 Asirvatham, Jennifer A Mears BS, Samuel J Asirvatham MD. Combined Auditory and Tragal Stimulation Headphone Effectively Increases Vagal Tone and Improves Short-Term Cognition. Biomed J Sci \& Tech Res 13(1)-2019. BJSTR. MS.ID.002351. DOI: 